\title{
Stopped-flow Light Scattering Analysis of Red Blood Cell Glycerol Permeability
}

Patrizia Gena ${ }^{1, *}$, Piero Portincasa ${ }^{2}$, Sabino Matera ${ }^{1}$, Yonathan Sonntag ${ }^{3}$, Michael Rützler ${ }^{3,4}$ and

Giuseppe Calamita ${ }^{1}$

1Department of Biosciences, Biotechnologies and Biopharmaceutics, University of Bari "Aldo Moro", Bari, Italy; ${ }^{2}$ Clinica Medica "A. Murri", Department of Biomedical Sciences and Human Oncology, Medical School, University of Bari "Aldo Moro", Bari, Italy; ${ }^{3}$ Department of Biochemistry and Structural Biology, Lund University, Lund, Sweden; ${ }^{4}$ ApoGlyx AB, Malmö, Sweden

*For correspondence: annapatrizia.gena@uniba.it

[Abstract] Stopped-Flow Light Scattering (SFLS) is a method devised to analyze the kinetics of fast chemical reactions that result in a significant change of the average molecular weight and/or in the shape of the reaction substrates. Several modifications of the original stopped-flow system have been made leading to a significant extension of its technical applications. One of these modifications allows the biophysical characterization of the water and solute permeability of biological and artificial membranes.

Here, we describe a protocol of SFLS to measure the glycerol permeability of isolated human red blood cells (RBCs) and evaluate the pharmacokinetics properties (selectivity and potency) of isoform-specific inhibitors of AQP3, AQP7 and AQP9, three mammalian aquaglyceroporins allowing transport of glycerol across membranes. Suspensions of RBCs (1\% hematocrit) are exposed to an inwardly directed gradient of $100 \mathrm{mM}$ glycerol in a SFLS apparatus at $20^{\circ} \mathrm{C}$ and the resulting changes in scattered light intensity are recorded at a monochromatic wavelength of $530 \mathrm{~nm}$ for $120 \mathrm{~s}$. The SFLS apparatus is set up to have a dead time of $1.6-\mathrm{ms}$ and $99 \%$ mixing efficiency in less than $1 \mathrm{~ms}$. Data are fitted to a single exponential function and the related time constant ( $\tau$, seconds) of the cell-swelling phase of light scattering corresponding to the osmotic movement of water that accompanies the entry of glycerol into erythrocytes is measured. The coefficient of glycerol permeability $\left(P_{g l y}, \mathrm{~cm} / \mathrm{s}\right)$ of RBCs is calculated with the following equation:

$$
P_{g l y}=1 /[(S / V) \tau]
$$

where $\tau(\mathrm{s})$ is the fitted exponential time constant and $S / V$ is the surface-to-volume ratio $\left(\mathrm{cm}^{-1}\right)$ of the analyzed RBC specimen. Pharmacokinetics of the isoform-specific inhibitors of AQP3, AQP7 and AQP9 are assessed by evaluating the extent of RBC $P_{g / y}$ values resulting after the exposure to serial concentrations of the blockers.

Keywords: Stopped-flow light scattering, Erythrocytes, Glycerol membrane permeability, Aquaglyceroporins, Aquaporin inhibitors 
[Background] The movement of water and solutes across biological and artificial membranes can be analyzed using different approaches. Assessing the rate with which the volume of any sealed particle lined by a membrane changes following the osmotically-driven movement of water represents an indirect approach widely used to measure biophysically the water and solute permeability of the particle specimen.

The Stopped-Flow Light Scattering (SFLS) method was devised to analyze the kinetics of rapid enzymatic reactions accompanied by significant changes of the molecular weight and/or shape of the reacting substrates (Riesner and Buenemann, 1973). SFLS soon proved to be an implementable, reliable and reproducible spectroscopic technique with a number of advantages and only few limitations. While the original method remains extensively used to study the chemical kinetics of fast reactions in solution the conventional SFLS apparatus has been modified to allow the measurement of the coefficient of solute and osmotic water permeability $\left(P_{\mathrm{s}}\right.$ and $P_{\mathrm{f}}$, respectively; $\left.\mathrm{cm} / \mathrm{s}\right)$ of both biological and artificial membranes (Terwilliger and Solomon, 1981; Mlekoday et al., 1983; van Heeswijk and van Os, 1986). SFLS is largely employed to assess the $P_{\mathrm{s}}$ and $P_{\mathrm{f}}$ of whole cells (Yang and Verkman, 2002; Maggio et al., 2018), organelles, extracellular vesicles (Calamita et al., 2005 and 2006; Ivanova et al., 2008; Soria et al., 2010), and vesicles of any kind of biological membrane (Shi et al., 1990; Calamita et al., 2012; Gena et al., 2017; Miyazawa et al., 2018). The method is also used to measure the permeability of artificial membranes such as liposomes (Zeidel et al., 1992 and 1994; Harris et al., 1994; Agre et al., 1999; Müller-Lucks et al., 2013) and polymersomes (Erbakan et al., 2014; Loo et al., 2017). SFLS is also employed to evaluate the Arrhenius activation energy $\left(E_{\mathrm{a}} ; \mathrm{kcal} / \mathrm{mol}\right)$ of water and solute transport based on the temperature dependence with which molecules cross the membrane (Agre et al., 1999; Yang and Verkman, 2002; Mathai et al., 2001; Mouro-Chanteloup et al., 2010).

Here, we describe a protocol of SFLS to measure the glycerol permeability of isolated human RBCs in presence or absence of three isoform-specific blockers of AQP3, AQP7 and AQP9 (Jelen et al., 2011; Sonntag et al., 2019), three aquaglyceroporin channels conducting glycerol, water and some other molecules. AQP3, AQP7 and AQP9 are variously expressed in the body playing roles both in health and disease (Yang et al., 2001; Hara-Chikuma and Verkman, 2006) and triggering strong pharmacological interest (Calamita et al., 2018). RBC glycerol permeability is measured after their exposure to different concentrations of the three blockers (vs. control RBCs treated with the vehicle alone). Useful information is acquired regarding the efficiency, selectivity and potency of the compounds in blocking the Aquaglyceroporin-facilitated glycerol transport as well as their preclinical sustainability (solubility, toxicity) for drug developments.

\section{Materials and Reagents}

1. Fifteen $\mathrm{ml}$ conical tubes (Falcon ${ }^{\mathrm{TM}}$ ) (Fisher Scientific, catalog number: 14-959-53A)

2. Sterile individually packaged $5 \mathrm{ml}$ pipettes (SARSTEDT, catalog number: 86.1253 .001 )

3. BD Vacutainer spray coated $\mathrm{K}_{2}$ EDTA tubes (BD, catalog number: 366643 )

4. Fresh human whole blood from healthy donors 
Collected following standard clinical procedures. The blood is drawn by direct puncture to the human vein located in the antecubital area of the arm and collected in in $\mathrm{K}_{2}$ EDTA tubes. After washings in DPBS, keep the blood at room temperature and proceed directly to stopped-flow light scattering measurements.

5. HTS13286 [AQP9 inhibitor (Jelen et al., 2011; Sonntag et al., 2019)], 2-[(4-Methyl-5-pyridin-4-yl-1,2,4-triazol-3-yl)sulfanyl]-N-(5-thiophen-2-yl-1,3,4-thiadiazol-2-yl) acetamide, MolPort, MolPort-002-839-693, stable, storage $2.5 \mathrm{mM}$ stock solutions dissolved in DMSO at $-20^{\circ} \mathrm{C}$. After freezing, warm well $\left(15 \mathrm{~min}\right.$ at $37^{\circ} \mathrm{C}$ and vortex) before use

6. DFP00173 [(AQP3 inhibitor (Sonntag et al., 2019)], 1-(2,6-dichlorophenyl)-3-(5-nitrothiophen-3-yl) urea, MolPort, MolPort-002-897-426, stable, storage $2.5 \mathrm{mM}$ stock solutions dissolved in DMSO at $-20^{\circ} \mathrm{C}$. After freezing, warm well ( $15 \mathrm{~min}$ at $37^{\circ} \mathrm{C}$ and vortex) before use

7. Z433927330 [(AQP7 inhibitor (Sonntag et al., 2019)], ethyl 4-(3-(4-(1H-pyrazol-1-yl)benzyl)ureido) benzoate, ENAMINE, MolPort-002-897-426, stable, storage $2.5 \mathrm{mM}$ stock solutions dissolved in DMSO at $-20^{\circ} \mathrm{C}$. After freezing, warm well $(15 \mathrm{~min}$ at $37^{\circ} \mathrm{C}$ and vortex) before use

8. Dulbecco's Phosphate Buffer Saline (DPBS) (EuroClone SpA, catalog number: ECB4004L), storage at $4{ }^{\circ} \mathrm{C}$; shelf-life: 24 months from date of manufacture

9. Glycerol (Sigma-Aldrich, catalog number: G7757), very stable, storage at room temperature, shelf-life: 3 to 5 years from date of manufacture

10. Dimethyl Sulfoxide (DMSO) (Sigma-Aldrich, catalog number: D8418), very stable, storage at room temperature in a dry place, since DMSO is known to be hygroscopic, shelf-life: 3 to 5 years from date of manufacture

11. Hypertonic solution consisting of DPBS added of glycerol (final osmolarity: $500 \mathrm{mOsM}$; final volume: $50 \mathrm{ml}$ ) (see Recipes)

\section{Equipment}

1. Refrigerate benchtop centrifuge (Thermo Fisher Scientific, Heraeus, model: Multifuge 1L-R, catalog number: 75004330)

2. Osmometer (EliTech, model: VAPRO Vapor Pressure Osmometer, catalog number: 5600)

3. Stopped-Flow Module (BioLogic Science Instruments, model: SFM20)

4. Spectrophotometer (Jasco EUROPE Srl, model: FP 6200)

5. P20 pipetman (Gilson, Fisher Scientific, catalog number: F123600)

6. P200 pipetman (Gilson, Fisher Scientific, catalog number: F123601)

7. P1000 pipetman (Gilson, Fisher Scientific, catalog number: F1236012)

8. Bibbyjet pipette controller (Bibby Sterilin Ltd, catalog number: PC1002)

9. Block heater (Stuart Scientific, catalog number: SBH130D)

10. Maxi Mixer Vortex 714 (Hosmotic Srl, catalog number: 29970003) 
11. $10 \mathrm{ml}$ Gastight Hamilton Syringes for SFM-20 (Jasco EUROPE Srl, catalog number: 950-12/3)

\section{Software}

1. Bio-Kine 32 software (BioLogic Science Instruments, available here)

2. GraphPad Prism 5.0 software (GraphPad Software, Inc., www.graphpad.com)

\section{Procedure}

A. Isolation of human RBCs

1. Collect a sample of human venous blood in a test-tube with anticoagulant (BD Vacutainer spray coated $\mathrm{K}_{2}$ EDTA tube).

2. Dilute the human venous blood sample 1:10 with Dulbecco's phosphate buffered saline (DPBS).

3. Wash the human venous blood sample for three times in DPBS $\left(800 \times \mathrm{g}\right.$ for $10 \mathrm{~min}$ at $\left.4{ }^{\circ} \mathrm{C}\right)$ to remove serum and cellular buffy coat.

4. Resuspend the resulting pellet of RBCs in DPBS to attain $1 \%$ hematocrit.

B. Assessment of RBCs glycerol permeability by SFLS

1. Prepare two solutions: $A)$ isotonic solution of $1 \times$ DPBS $(300 \mathrm{mOsM})$ and $B)$ hypertonic solution consisting of $1 \times$ DPBS added with glycerol (final osmolarity: $500 \mathrm{mOsM}$ ).

2. Verify the osmolarity of the above reported solutions by using an osmometer. Mixing of solutions $A$ and $B$ will result in a $100 \mathrm{mM}$ inwardly direct gradient of glycerol.

3. Set up the stopped-flow light scattering device (Figure 1) as follows:

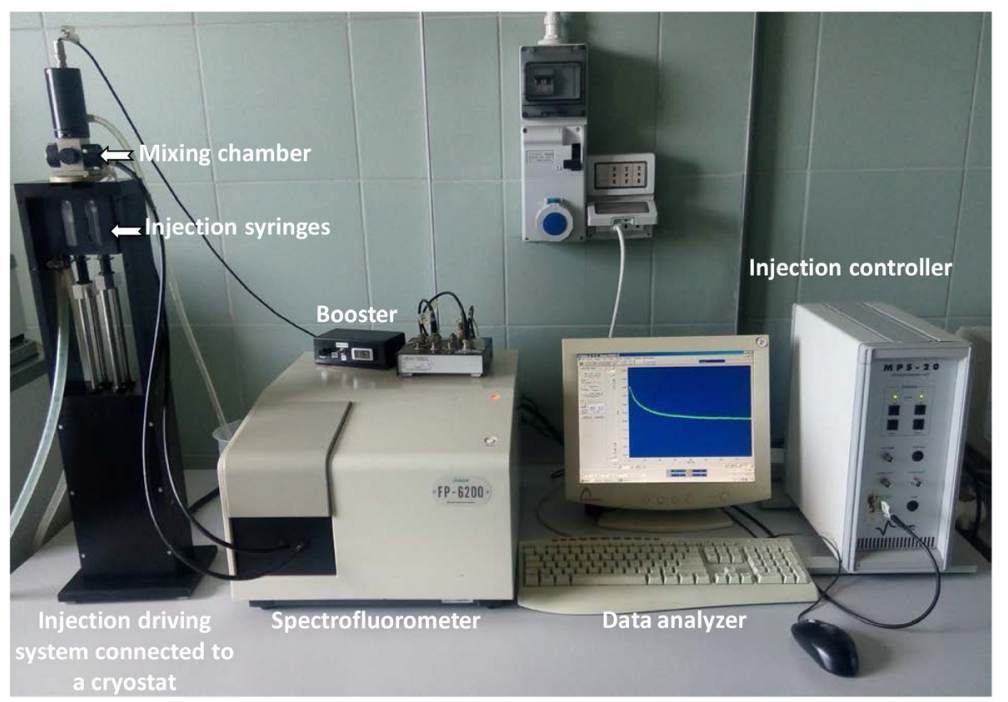

Figure 1. Stopped-flow light scattering apparatus. The SFM-20 injection system coupled to a spectrophotometer is an ideal system for $90^{\circ}$ light scattering assays. In this protocol, the 
SFLS apparatus is employed for the assessment of erythrocyte glycerol permeability. The SFM-20 injection driving motor triggers the syringe pistons allowing the solutions to pass simultaneously into the mixing chamber directly connected to an observation cell irradiated with a xenon source lamp. With the SFM-20's driving control of the total flow rate shear forces are minimized. A synchronized electro-valve stops the sample flow and prevents the occurrence of overpressure artifacts. All the data collected by a detector are then analyzed by the software Bio-Kine 32.

a. Set the cryostat temperature at $20^{\circ} \mathrm{C}$;

b. Initialize the injection system and the spectrophotometer by the MPS-20 and Bio-Kine 32 software, respectively;

c. Set the SFLS apparatus using the following MPS-20 parameters (Figure 2):

i. Dead time (time required for the solutions to flow from the mixing chamber to the observation cell): $1.6 \mathrm{~ms}$;

ii. $99 \%$ mixing efficiency in $<1 \mathrm{~ms}$;

iii. Mixing time: $20 \mathrm{~ms}$;

iv. Volume injected by syringe 1: $100 \mu$;

v. Volume injected by syringe 2: $100 \mu$;

vi. Set the excitation and emission wavelengths on the spectrophotometer to $530 \mathrm{~nm}$. The bandwidth of excitation and emission must be set to $20 \mathrm{~nm}$.

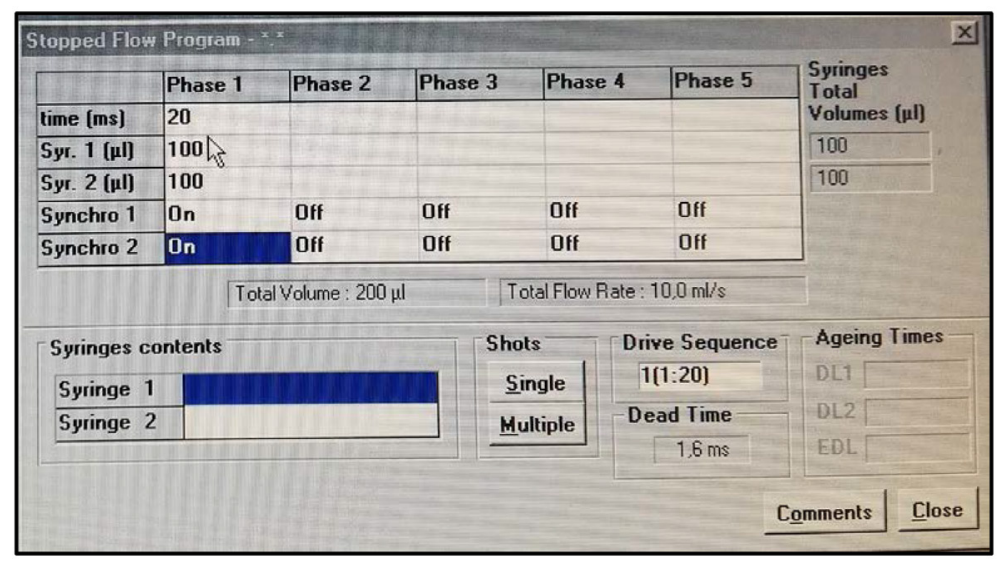

Figure 2. SFM-20 software for single mixing applications. SFM-20 is set up for rapid and easy SFLS experimental design. The user sets the mixing ratio, the size of syringes and cuvette, and the volume samples. Color-coded windows display calculated values and alert the user to out of range experimental conditions. The estimated dead time is automatically displayed, and a pretrigger is also available to be sure the stationary state is reached accurately.

4. Equilibrate $15 \mu \mathrm{l}$ of RBCs suspension in $2.5 \mathrm{ml}$ of isotonic DPBS for $5 \mathrm{~min}$ and then fill one of the two syringes with the cell suspension. 
5. Fill the other syringe with the hypertonic solution (DPBS added of glycerol, final osmolarity: 500 mOsM).

6. Inject the erythrocyte suspension together with the hypertonic solution (osmotic gradient, 100 mOsM) into a mixing chamber. As a result of a glycerol gradient, at first, a rapid water outflow occurs leading to cell shrinkage and causing an increase in scattered light intensity (I) over time (s) (Figure 3), subsequently, as a consequence of the glycerol entry, water flows into the cells causing cell swelling whose kinetics is displayed as decrease in scattered light intensity (I) over time (s) (Figure 3).

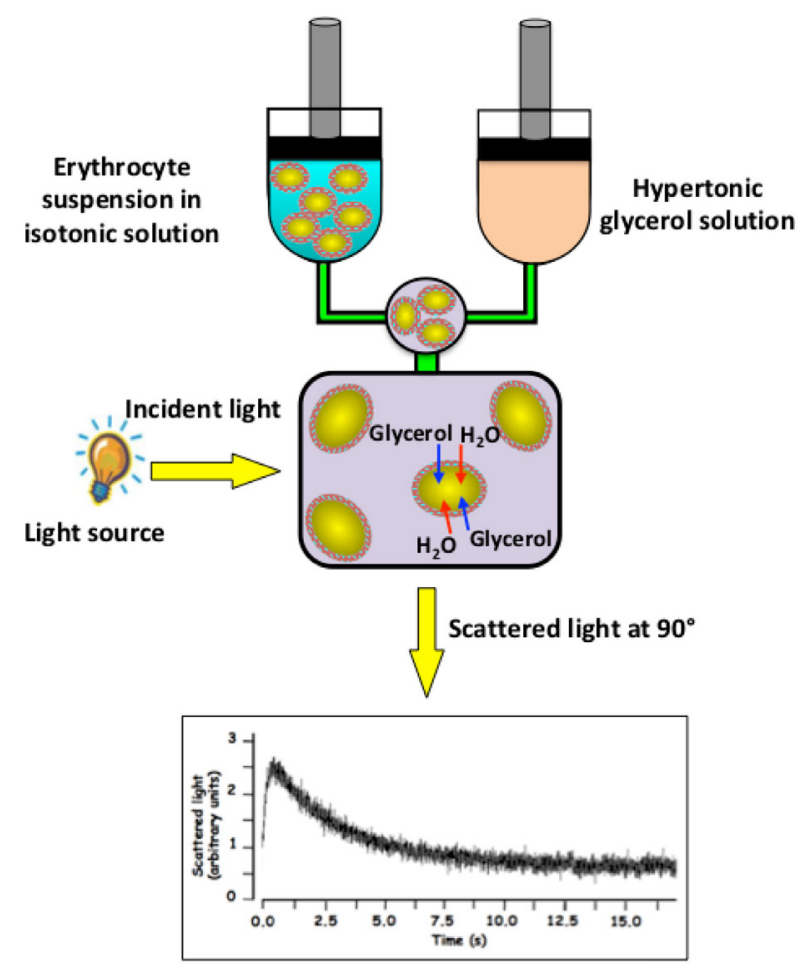

Figure 3. Stopped-flow light scattering measurements of erythrocyte glycerol permeability. The addition of extracellular glycerol causes a rapid increase in the intensity of scattered light due to the water efflux induced by the osmotic gradient (shrinkage) followed by a slower scattered light decrease (swelling) induced by the osmotic influx of water the accompanies the entry of glycerol into the cells. Glycerol permeability is calculated by measuring the exponential time constant $(\tau, s)$ fitted to the cell swelling phase of the $90^{\circ}$ light scattering time course associated to the influx of glycerol and water.

7. By using the Bio-Kine 32 software, fit the data to a single exponential function and measure the time rate constant $(\tau, s)$ of the cell swelling phase during the entry phase of glycerol and water into the erythrocytes (Figure 4). 


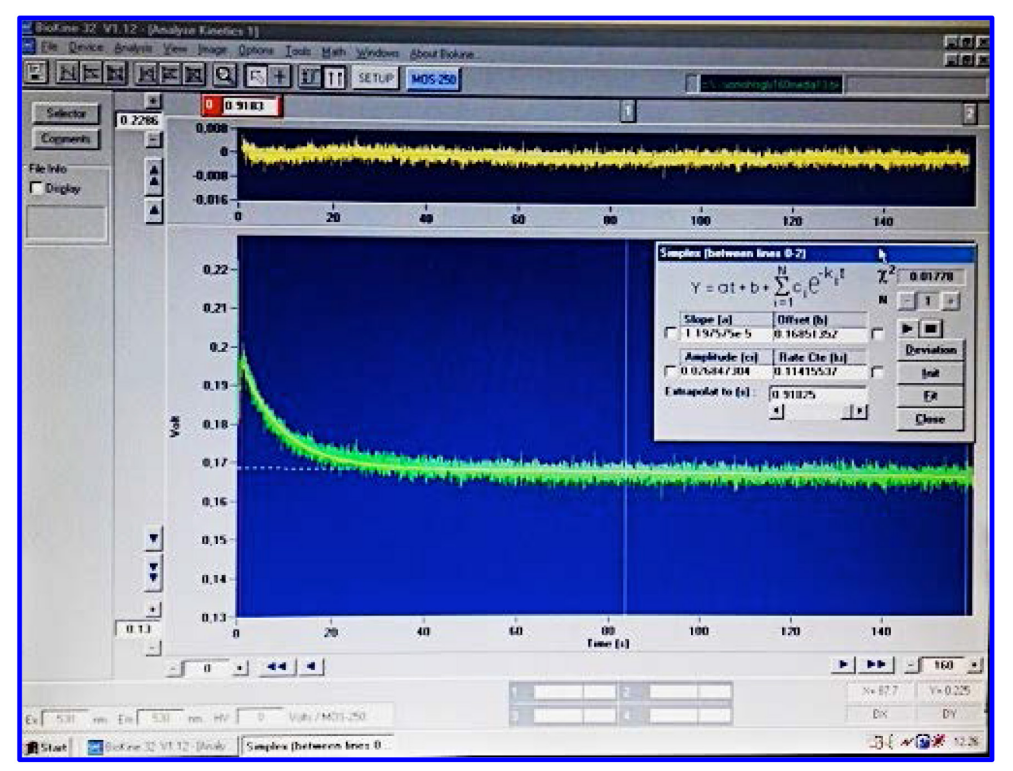

Figure 4. Bio-Kine 32 for data acquisition and analysis. Acquisition and analysis of signals produced by SFLS device are carried out using the Bio-Kine 32 software. Bio-Kine 32 has been designed to complement the SFM-20 software and to connect the spectrometer to the mixing device.

8. For the inhibition studies (Figure 5), dissolve the compounds in DMSO as previously reported, add the right volume of inhibitor solution to the erythrocyte suspension and incubate for $10 \mathrm{~min}$ at $20{ }^{\circ} \mathrm{C}$ prior to the measurements of light scattering. The final concentration of HTS13286, DFP00173 and Z433927330 is $25 \mu \mathrm{M}$ in $1 \%$ DMSO. Carry out all the inhibition assays while keeping the final concentration of DMSO constant at $1 \%$. To keep constant the concentration of DMSO needed for inhibition studies, prepare a series of DMSO solutions of the drug with different concentrations, and do the same dilution with the buffer solution, in this way is possible to get buffer solutions of different drug concentrations while maintaining the same percentage of DMSO. 

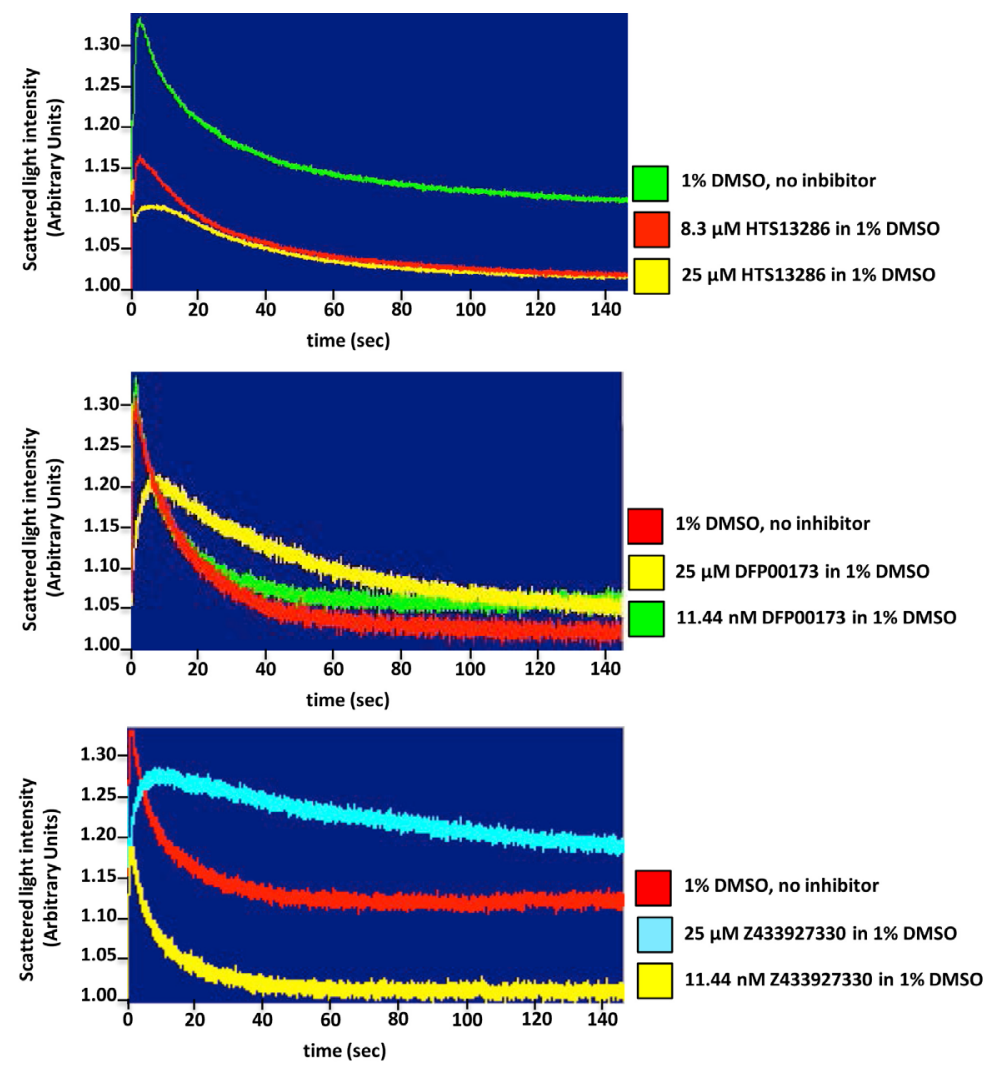

Figure 5. Inhibition studies of glycerol transport across erythrocyte plasma membrane. Stopped-flow light scattering technique is a valuable tool for assessing water and solute movement across biological membranes and the characterization of pharmacokinetic properties (efficiency, specificity, potency) of protein channels and carrier inhibitors, as well. Representative tracings of glycerol permeation into human red blood control cells (no inhibitor) or incubated for 10 min with two different concentrations of each inhibitor, one blocking mostly the glycerol-facilitated diffusion and the other being ineffective. A significant inhibition of glycerol permeability is seen adding $25 \mu \mathrm{M}$ of all three inhibitors, HTS 13286 (A), DFP00173 (B) and Z433927220 (C) compared to the control cells (1\% DMSO).

9. RBCs suspensions incubated with $1 \%$ DMSO (solvent of all the three inhibitors) (10 min at $20^{\circ} \mathrm{C}$ as well as the experimental conditions) represent the control condition.

C. Calculation of the glycerol permeability coefficient $\left(P_{\mathrm{gly}}, \mathrm{cm} / \mathrm{s}\right)$.

The osmotic water influx following glycerol entry into the erythrocytes and therefore the resulting swelling leads to a decrease of the scattered light intensity over time (Figure 2). Calculate the glycerol permeability coefficient $\left(P_{g l y}, \mathrm{~cm} / \mathrm{s}\right)$ by measuring the $\tau$ value (using the Bio-Kine 32 software), exponential time constant fitted to the data related to the cell swelling phase of the curve, and using the following equation:

$$
P_{g l y}=1 /[(S / V) \tau]
$$


where $S / V$ is the surface-to-volume ratio of the analyzed RBCs.

A practical example for the calculation of the $P_{g l y}$ of the RBC plasma membrane is described below:

$$
P_{g l y}=1 /[(\mathrm{S} / \mathrm{V}) \tau]
$$

where S/V is the initial surface/volume ratio of RBCs. Assuming that the cell shape is roughly spherical and the cell outer surface is regular and completely smooth, S/N may be obtained as follows:

$$
S / V=4 \pi r^{2} /(4 / 3) \pi r^{3}=3 / r
$$

where $r$ is the cell radius.

The above $P_{\text {gly }}$ equation can now be also written as follows:

$$
P_{\text {gly }}=1 /(3 / r) \tau=r / 31 / \tau
$$

The RBC mean radius is of $4 \times 10^{-5} \mathrm{~cm}$ and the $\tau$ value corresponds to the reciprocal of a time constant, $K_{\mathrm{i}}\left(\mathrm{s}^{-1}\right)$, calculated from the exponential fitting of the SFLS traces $\left(\tau_{\mathrm{RBC}}=1 / K_{\mathrm{i}}(\mathrm{RBC})=\right.$ $\left.1 / 0.110 \mathrm{~s}^{-1}=9.0 \mathrm{~s}\right)$. The $P_{g l y}$ value is then calculated as follows:

$$
P_{\text {gly }}=4 \times 10^{-5} \mathrm{~cm} / 31 / 9.0 \mathrm{~s}=1.48 \times 10^{-6} \mathrm{~cm} / \mathrm{s}
$$

\section{Data analysis}

Stopped-flow light scattering experiments should be performed at least in triplicate. All data resulted from three to five different human red blood cell suspensions are expressed as mean \pm SEM. Data are analyzed statistically by two-way ANOVA in GraphPad Prism 5.0 and the results are considered statistically significant when $P<0.05$.

\section{$\underline{\text { Recipes }}$}

1. Hypertonic solution consisting of PBS added of glycerol (final osmolarity: $500 \mathrm{mOsM}$; final volume: $50 \mathrm{ml}$ )

Add $0.730 \mathrm{ml}$ of $100 \%$ glycerol to $1 \times$ DPBS and mix gently until complete mixing. 


\section{Acknowledgments}

Financial support to GC from Italian "Programmi di Ricerca Scientifica di Rilevante Interesse Nazionale 2017" (PRIN2017; grant \# 2017J92TM5) and to YS from Stiftelsen Olle Engkvist Byggmästare is gratefully acknowledged.

The protocol object of the present article is derived from a previous research paper from our laboratories (Sonntag et al., 2019).

\section{Competing interests}

The authors declare no conflict of interest.

\section{Ethics}

All human subjects provided written informed consent for participation in the study, in accordance with ICH/GCP guidelines. The protocol was approved by the local ethics committee (authorization \# FMF01-5870-27-02-2019).

\section{References}

1. Agre, P., Mathai, J. C., Smith, B. L. and Preston, G. M. (1999). Functional analyses of aquaporin water channel proteins. Methods Enzymol 294: 550-572.

2. Calamita, G., Ferri, D., Gena, P., Liquori, G. E., Cavalier, A., Thomas, D. and Svelto, M. (2005). The inner mitochondrial membrane has aquaporin-8 water channels and is highly permeable to water. J Biol Chem 280(17): 17149-17153.

3. Calamita, G., Gena, P., Meleleo, D., Ferri, D. and Svelto, M. (2006). Water permeability of rat liver mitochondria: A biophysical study. Biochim Biophys Acta 1758(8): 1018-1024.

4. Calamita, G., Gena, P., Ferri, D., Rosito, A., Rojek, A., Nielsen, S., Marinelli, R. A., Fruhbeck, G. and Svelto, M. (2012). Biophysical assessment of aquaporin-9 as principal facilitative pathway in mouse liver import of glucogenetic glycerol. Biol Cell 104(6): 342-351.

5. Calamita, G., Perret, J. and Delporte, C. (2018). Aquaglyceroporins: Drug Targets for Metabolic Diseases? Front Physiol 9: 851.

6. Erbakan, M., Shen, Y. X., Grzelakowski, M., Butler, P. J., Kumar, M. and Curtis, W. R. (2014). Molecular cloning, overexpression and characterization of a novel water channel protein from Rhodobacter sphaeroides. PLoS One 9(1): e86830.

7. Gena, P., Buono, N. D., D'Abbicco, M., Mastrodonato, M., Berardi, M., Svelto, M., Lopez, L. and Calamita, G. (2017). Dynamical modeling of liver Aquaporin-9 expression and glycerol permeability in hepatic glucose metabolism. Eur J Cell Biol 96(1): 61-69. 
8. Hara-Chikuma, M. and Verkman, A. S. (2006). Physiological roles of glycerol-transporting aquaporins: the aquaglyceroporins. Cell Mol Life Sci 63(12): 1386-1392.

9. Harris, H. W., Jr., Zeidel, M. L., Jo, I. and Hammond, T. G. (1994). Characterization of purified endosomes containing the antidiuretic hormone-sensitive water channel from rat renal papilla. J Biol Chem 269(16): 11993-12000.

10. Ivanova, E., Jowitt, T. A. and Lu, H. (2008). Assembly of the mitochondrial Tim9-Tim10 complex: a multi-step reaction with novel intermediates. J Mol Biol 375(1): 229-239.

11. Jelen, S., Wacker, S., Aponte-Santamaria, C., Skott, M., Rojek, A., Johanson, U., Kjellbom, P., Nielsen, S., de Groot, B. L. and Rützler, M. (2011). Aquaporin-9 protein is the primary route of hepatocyte glycerol uptake for glycerol gluconeogenesis in mice. J Biol Chem 286(52): 44319-44325.

12. Loo, S. L., Siti, W., Thiyagarajan, M., Torres, J., Wang, R. and Hu, X. (2017). Reproducible preparation of proteopolymersomes via sequential polymer film hydration and membrane protein reconstitution. Langmuir 33(43): 12336-12343.

13. Maggio, A., Bernardino, R. L., Gena, P., Alves, M. G., Oliveira, P. F. and Calamita, G. (2018). $\underline{A}$ stopped-flow light scattering methodology for assessing the osmotic water permeability of whole sertoli cells. Methods Mol Biol 1748: 279-286.

14. Mathai, J. C., Sprott, G. D. and Zeidel, M. L. (2001). Molecular mechanisms of water and solute transport across archaebacterial lipid membranes. J Biol Chem 276(29): 27266-27271.

15. Miyazawa, Y., Mikami, S., Yamamoto, K., Sakai, M., Saito, T., Yamamoto, T., Ishibashi, K. and Sasaki, S. (2018). AQP2 in human urine is predominantly localized to exosomes with preserved water channel activities. Clin Exp Nephrol 22(4): 782-788.

16. Mlekoday, H. J., Moore, R. and Levitt, D. G. (1983). Osmotic water permeability of the human red cell. Dependence on direction of water flow and cell volume. J Gen Physiol 81(2): 213-220.

17. Mouro-Chanteloup, I., Cochet, S., Chami, M., Genetet, S., Zidi-Yahiaoui, N., Engel, A., Colin, Y., Bertrand, O. and Ripoche, P. (2010). Functional reconstitution into liposomes of purified human RhCG ammonia channel. PLoS One 5(1): e8921.

18. Müller-Lucks, A., Gena, P., Frascaria, D., Altamura, N., Svelto, M., Beitz, E. and Calamita, G. (2013). Preparative scale production and functional reconstitution of a human aquaglyceroporin (AQP3) using a cell free expression system. N Biotechnol 30(5): 545-551.

19. Riesner, D. and Buenemann, H. (1973). A stopped-flow apparatus with light-scattering detection and its application to biochemical reactions. Proc Natl Acad Sci USA 70(3): 890-893.

20. Shi, L. B., Wang, Y. X. and Verkman, A. S. (1990). Regulation of the formation and water permeability of endosomes from toad bladder granular cells. J Gen Physiol 96(4): 789-808.

21. Sonntag, Y., Gena, P., Maggio, A., Singh, T., Artner, I., Oklinski, M. K., Johanson, U., Kjellbom, P., Nieland, J. D., Nielsen, S., Calamita, G. and Rutzler, M. (2019). Identification and characterization of potent and selective aquaporin-3 and aquaporin-7 inhibitors. $J$ Biol Chem 294(18): 7377-7387. 
22. Soria, L. R., Fanelli, E., Altamura, N., Svelto, M., Marinelli, R. A. and Calamita, G. (2010). Aquaporin-8-facilitated mitochondrial ammonia transport. Biochem Biophys Res Commun 393(2): 217-221.

23. Terwilliger, T. C. and Solomon, A. K. (1981). Osmotic water permeability of human red cells. $J$ Gen Physiol 77(5): 549-570.

24. van Heeswijk, M. P. and van Os, C. H. (1986). Osmotic water permeabilities of brush border and basolateral membrane vesicles from rat renal cortex and small intestine. $J$ Membr Biol 92(2): 183-193.

25. Yang, B., Ma, T. and Verkman, A. S. (2001). Erythrocyte water permeability and renal function in double knockout mice lacking aquaporin-1 and aquaporin-3. J Biol Chem 276(1): 624-628.

26. Yang, B. and Verkman, A. S. (2002). Analysis of double knockout mice lacking aquaporin-1 and urea transporter UT-B. Evidence for UT-B-facilitated water transport in erythrocytes. $J$ Biol Chem 277(39): 36782-36786.

27. Zeidel, M. L., Ambudkar, S. V., Smith, B. L. and Agre, P. (1992). Reconstitution of functional water channels in liposomes containing purified red cell CHIP28 protein. Biochemistry 31(33): 7436-7440.

28. Zeidel, M. L., Nielsen, S., Smith, B. L., Ambudkar, S. V., Maunsbach, A. B. and Agre, P. (1994). Ultrastructure, pharmacologic inhibition, and transport selectivity of aquaporin channel-forming integral protein in proteoliposomes. Biochemistry 33(6): 1606-1615. 